\title{
Étude de la relation entre le climat de la classe et la satisfaction des étudiants dans un contexte universitaire
}

\section{GANDAYI GABUDISA BUSUGUTSALA}

\begin{abstract}
RÉSUMÉ
Cet article présente les résultats partiels d'une étude globale de la relation entre le climat de la classe et l'apprentissage dans un contexte universitaire. L'apprentissage y est considéré comme un processus complexe d'engagement, de participation et de développement intégral des étudiants qui se réalise dans un contexte riche de rôles et d'interactions. Nous ne présentons ici que les résultats d'un seul des indicateurs de l'apprentissage retenus pour fins d'opérationalisation: la satisfaction des étudiants. Ces résultats confirment, d'une part, qu'il existe une relation entre le climat d'apprentissage et la variation de la satisfaction des étudiants. D'autre part, le climat d'apprentissage varie, comme d'ailleurs son influence directe ou en tant que variable médiatrice entre les caractéristiques des étudiants et des professeurs* et l'apprentissage.
\end{abstract}

* Le masculin pluriel désigne aussi bien les hommes que les femmes; il est utilisé pour alléger le texte et sans intention discriminatoire. 


\section{ABSTRACT}

This article presents partial results of a global study about the relation between the class climate and the learning in a university context. Learning is a complex process of involvement and total development of the students. This happens in a rich environment of roles and interactions. We are presenting one of the learning indicators: the students' satisfaction. On one hand, results confirm that there is a relation between the class climate the students' varied levels of satisfaction. On the other hand, the class climate varies like its direct influence or as a variable mediator between the students' and the professors' characteristics versus the learning process.

\section{PROBLÉMATIQUE}

Réinterprétant les données du Rapport Coleman (1966), Hanushek (1972), parmi d'autres chercheurs, a constaté que des élèves possédant des caractéristiques similaires quant aux antécédents familiaux, au statut socio-économique et aux résultats scolaires antérieurs, et fréquentant la même école vivaient des expériences d'éducation différentes simplement du fait qu'ils étaient dans différentes classes. Il en conclut que les classes à l'intérieur d'une même école sont un facteur déterminant des variations dans l'apprentissage des élèves. Il préconise, en conséquence, que la recherche de politiques efficaces en éducation déplacent leur centre d'intérêt de l'école en général vers les classes prises individuellement.

De fait, dans la recherche actuelle d'indicateurs permettant d'évaluer les politiques en matière d'efficacité ou de qualité de l'enseignement, le niveau d'analyse a évolué passant d'une approche "macro" ou générale à une perspective "micro". De même, l'attention s'est déplacée des facteurs intrants (caractéristiques personnelles, économiques, etc.) vers la variable processus (situation d'enseignement-apprentissage ....) et les résultats ou produits de l'éducation.

La recension des écrits de 1975 à 1986 de Fraser (1986) indique que, sans être la seule variable, le climat de l'école et de la classe explique en partie la variation dans l'apprentissage en général et, en particulier, dans le rendement scolaire des étudiants, leur satisfaction, leur persévérance, leur confiance en soi, leur degré de socialisation, leurs attitudes face aux matières enseignées, leurs humeurs, leur image de soi et leur degré d'anxiété. 
Cependant, les travaux se sont centrés sur le climat de l'organisation scolaire entière, d'une part. D'autre part, les recherches sur le climat de la classe sont centrées sur les écoles primaires et secondaires; le niveau universitaire est resté un parent pauvre dans le monde et au Canada français en particulier. Aussi, il y a plus de trente ans, Pace (1961) avaitil déjà critiqué la recherche qui n'est pas assez centrée sur le climat de la classe au collège et à l'université et sa relation avec le rendement, la motivation et la satisfaction des étudiants. Des travaux récents (Carothers \& Sevingy, 1993; Gartner, 1993; Serey, 1993) suggèrent aussi d'orienter la recherche dans cette direction.

Le présent article rapporte des résultats d'une étude globale qui se situe dans la foulée des préoccupations actuelles des théoriciens et des praticiens de l'éducation cherchant à comprendre les phénomènes d'abandon et de réussite ou d'échec scolaires ainsi que d"'imputabilité" ou l'obligation des éducateurs et des systèmes éducatifs de rendre des comptes de leur efficience et de leur efficacité en raison des ressources publiques investies dans l'éducation.

\section{OBJECTIFS DE LA RECHERCHE}

Cette étude vise deux objectifs: 1) explorer l'existence et la nature de la relation entre le climat de la classe dans un contexte universitaire, et la satisfaction des étudiants; 2) comparer, d'une part, les perceptions du climat d'apprentissage, et d'autre part, leur influence sur la satisfaction des étudiants.

Deux questions de recherche simples formulées à partir de ces objectifs nous guideront:

1. Quelle relation existe-t-il entre le climat d'apprentissage ou de la classe, globalement et pour chacune de ses composantes, et la variation de la satisfaction des étudiants dans un contexte universitaire?

2. Quelle est l'influence des caractéristiques des étudiants et des professeurs sur la perception du climat d'apprentissage ou de la classe et sur la variation de la satisfaction des étudiants dans un contexte universitaire?

La première question résume le but principal de cette étude, à savoir la relation entre le climat de la classe à l'université et la satisfaction des étudiants. La seconde question annonce les comparaisons possibles à 
faire: il s'agit de montrer comment l'hétérogénéité des caractéristiques des étudiants et des professeurs, ainsi que la variation des climats des classes expliquent les différences dans la satisfaction.

\section{CADRE CONCEPTUEL}

Nous tenterons, dans cette section, de clarifier certaines notions clés et d'en dégager les éléments fondamentaux.

\section{Essai de définition du climat d'apprentissage ou de la classe}

Toutes les définitions ou descriptions du concept de climat organisationnel (Brunet, 1987; Fourgous \& Iturralde, 1991; Moos, 1979; Savoie, 1987; Tagiuri, 1968) renvoient non seulement aux réalités objectives qui constituent l'organisation, mais encore davantage à la perception entretenue par les individus de leur environnement ou de leur situation de travail en fonction de leur personnalité, de leurs attentes et de leurs besoins.

Les organisations scolaires ont été fortement marquées par les diverses théories de l'organisation. Ainsi, l'importance du climat organisationnel mise en lumière par plusieurs travaux a poussé des chercheurs en éducation à s'intéresser aux caractéristiques spécifiques du milieu scolaire dont la perception est susceptible d'avoir une influence. Du même coup, l'on distingue, d'une part, la perception référant à l'ensemble des attributs de l'organisation qui constitue le climat "organisationnel". L'influence de ce dernier s'exerce surtout sur la motivation des enseignants; son incidence sur l'apprentissage des étudiants est, par conséquent, indirecte (Anderson, 1982; Fraser \& Rentoul, 1982; Genn, 1984; Hoy \& Forsyth, 1986). D'autre part, la perception des caractéristiques de la classe constitue spécifiquement le climat d'apprentissage. Celui-ci "se veut relié de façon plus étroite au processus de production de connaissance et se prête plus facilement à une intervention pédagogique" (Michaud, Forgette-Giroux \& Richard, 1989, p. 28). Cette étude concerne spécifiquement le climat d'apprentissage ou de classe.

\section{Clarification de la notion de satisfaction}

Deux aspects de la satisfaction des étudiants sont habituellement abordés dans les études: la satisfaction "scolaire" ou directement liée à l'apprentissage, à l'enseignement et aux programmes d'études; et la 
satisfaction liée aux conditions de vie matérielles, financières et résidentielles du campus. Notre étude porte sur le premier aspect. Aitken (1982) en a proposé un modèle conceptuel intéressant dans lequel, sous forme d'équation mathématique, il la définit comme suit:

"Academic Satisfaction

(AS) $=$ bo+b1 AP+b2QC+b3QI+b4QAA +b5MS+b6P+ub": la satisfaction scolaire (AS) résulte du rendement ou performance de l'étudiant (AP), de la qualité du programme d'études (QC), de la qualité de l'enseignement (QI), de la qualité de l'encadrement scolaire ou pédagogique (QAA), de la satisfaction de l'étudiant par rapport à la discipline ou matière (MS), de la personnalité ou caractéristiques individuelles de l'étudiant $(\mathrm{P})$, et de bien d'autres facteurs "intercept et résidus" (bo, ub).

En résumé, les éléments suivants se dégagent de différentes définitions proposées et témoignent de la complexité des notions ou phénomènes de climat d'apprentissage ou de la classe et de la satisfaction des étudiants: l'importance accordée à la perception de l'individu; la référence à son rôle, à sa personnalité et à ses besoins; l'insistance sur la participation et l'engagement de l'individu à une coopération ou activité de groupe, ainsi que sur les moyens (processus) plutôt que sur la fin.

\section{MÉTHODOLOGIE}

\section{Modèle conceptuel}

Dans cette étude, nous avons adopté l'approche systémique selon laquelle l'ensemble des relations entre les variables retenues à l'intérieur du cadre structural de la classe s'insère dans l'organisation universitaire qui, à son tour, est un système ouvert, en interaction avec son environnement ou système social plus vaste. Ce modèle conceptuel prend, schématiquement, la configuration suivante (Figure 1).

\section{Instruments de mesure}

Le climat de la classe peut être diagnostiqué en observant la disposition des sièges, les mouvements physiques des étudiants, leurs expressions corporelles, leur perception des espaces, de l'éclairage, des équipements, etc. Pour le mesurer dans la présente étude, nous avons 


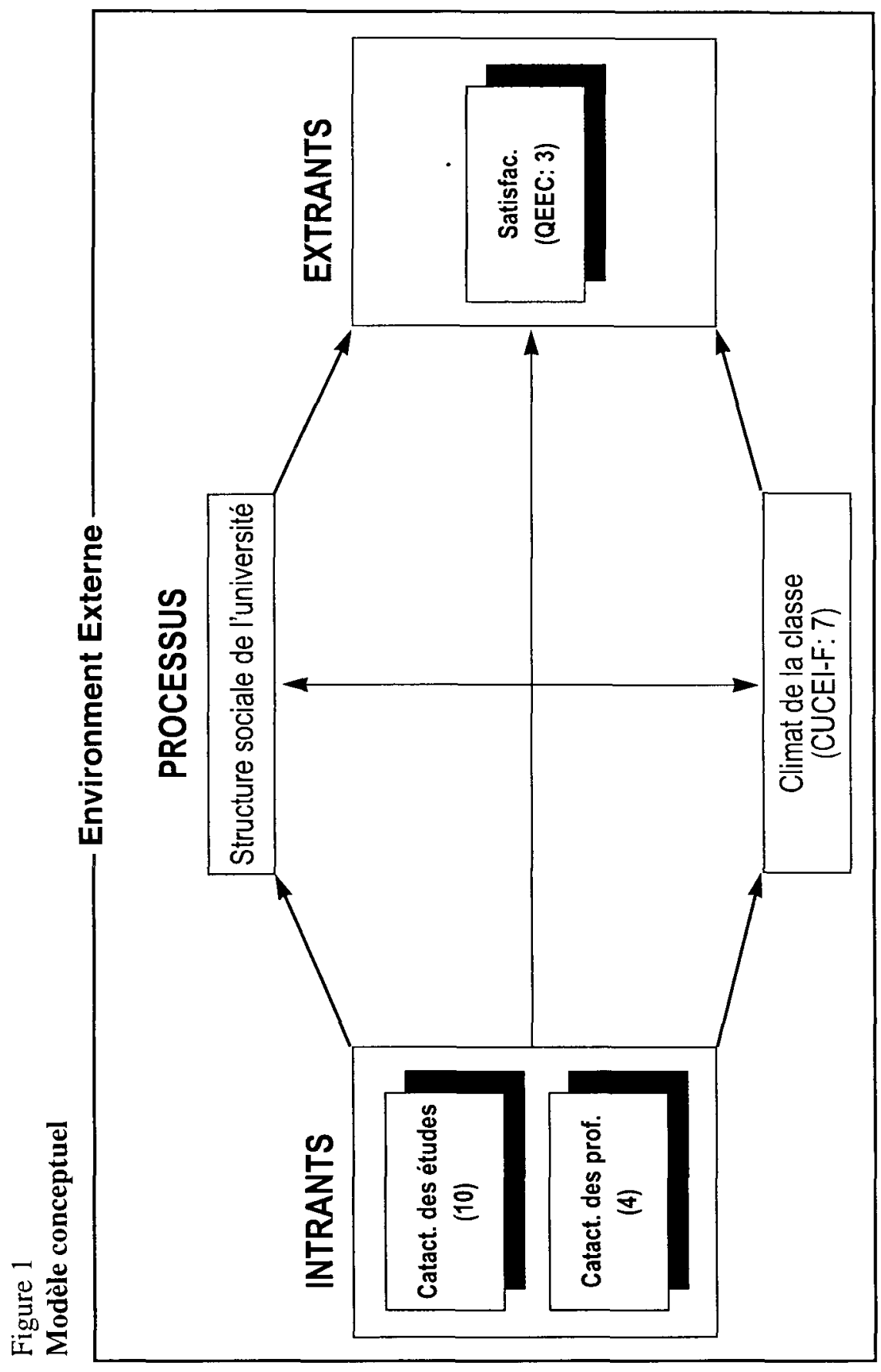

The Canadian Journal of Higher Education

Volume XXVIII, No. 2, 3, 1998 
utilisé la version française d'un instrument ad hoc mis au point par Fraser et ses collaborateurs (1986): le College and University Classroom Environment Inventory (CUCEI). Tout en étant court - sept échelles de sept items chacune, soit au total 49 énoncés - le CUCEI a l'avantage de mesurer les catégories importantes qui caractérisent tout environnement social (universités, hôpitaux, institutions correctionnelles, organisations militaires, familles, groupes sociaux et thérapeutiques, milieux de travail), à savoir les dimensions "personnalité, relation, et maintien et développement du système." En outre, le CUCEI ne restreint pas l'inventaire du climat d'apprentissage aux choix limités 'vrai ou faux' de certains autres questionnaires, il permet un choix de réponses graduées de type Likert à cinq niveaux.

La version française de cet instrument désormais dénommé CUCEI-F, de notre cru, a été validée en mars 1991 avec un échantillon de 390 étudiants de 14 classes de la Cité collégiale (Ottawa) et de l'Université d'Ottawa. Les résultats de .ce pré-test - non présentés en détail ici montrent que les coefficients alpha de Cronbach pour chacune des sept échelles du climat varient de 0,66 à 0,89 ; pour l'ensemble du test, l'indice global de fidélité (coefficient alpha de Cronbach) est de 0,90. Les moyennes de corrélation entre paires d'échelles se sont avérées suffisamment faibles pour que les échelles ne soient pas redondantes et suffisamment élevées pour confirmer la cohérence du test. Ces résultats de mise à l'épreuve des qualités métrologiques du CUCEI-F sont, d'ailleurs, dans l'ensemble, supérieurs à ceux qui ont été obtenus dans les validations de Fraser et ses collaborateurs (1986) et de Marcelo (1988).

Les sept dimensions du climat de la classe mesurées sont:

1. Personnalisation (Pers.): l'accent est mis sur les chances des étudiants d'interagir avec le professeur en classe, et sur l'intérêt porté par celui-ci au bien-être personnel des étudiants.

2. Participation (Par.): l'étendue de l'engagement et de la participation active et attentive des étudiants aux discussions en classe (au cours).

3. Cohésion du groupe (Coh.): l'étendue de la connaissance et du soutien mutuels ainsi que le niveau d'amitié et de solidarité que les étudiants ont entre eux.

4. Motivation (Mot.): le plaisir et la satisfaction que l'on éprouve lorsqu'on est en train d'apprendre et de se surpasser dans son travail d'étudiant. 
5. Intérêt pour la tâche (Int.): le niveau de clarté et de la bonne structuration des activités qui se font en classe; les aspects organisationnels tels que la qualité du plan de cours, les références suggérées, etc.

6. Innovation (Inn.): la quantité et la qualité des activités d'apprentissage nouvelles et novatrices que le professeur planifie; ses nouvelles méthodes d'enseignement et la nouveauté des tâches assignées aux étudiants en classe.

7. Individualisation (Ind.): la façon dont les étudiants sont associés au processus décisionnel et sont traités selon leurs différences individuelles d'habileté, d'intérêt et de rythme d'apprentissage.

La satisfaction des étudiants, quant à elle, a été mesurée à l'aide du "Questionnaire d'évaluation de l'enseignement et des cours" (QÉEC) de l'Université d'Ottawa. C'est un instrument standard qui compte 12 questions dont nous n'avons utilisé que les trois questions obligatoires portant respectivement sur les aptitudes du professeur en tant qu'enseignant (Apt), son efficacité à transmettre la matière (Eff) et le soin qu'il met à préparer ses cours (Prép).

Enfin, les données sur les variables démographiques ou intrants ont été obtenues à l'aide des questions élaborées à cette fin par l'auteur. Ces trois instruments faisaient partie d'un seul document de cueillette des données que l'on trouvera en annexe du présent article.

\section{PROCÉDURE}

Pour la cueillette des données, nous contactions les professeurs choisis au hasard. Nous avons procédé nous-mêmes à l'administration du questionnaire (constitué de questions sur les caractéristiques individuelles, le climat et la satisfaction) auquel les étudiants répondaient en salle de classe durant le temps du cours. Les professeurs étaient priés, chaque fois, de sortir de la classe.

\section{Échantillon}

L'échantillon est constitué de 39 classes, soit 984 étudiants, de langue française du premier cycle, choisies au hasard dans les facultés d'administration, des arts, des sciences et des sciences sociales de 
Tableau 1

Répartition par faculté des classes $(N=39)$ des étudiants $(N=984)$ constituant l'échantillon

\begin{tabular}{lccccc}
\hline Characteristiques & Administration & Arts & Sciences & $\begin{array}{c}\text { Sciences } \\
\text { sociales }\end{array}$ & Total \\
\hline Classes & 10 & 14 & 60 & 9 & 39 \\
\hline Étudiants & 326 & 278 & 161 & 219 & 984 \\
& $33,1 \%$ & $28,3 \%$ & $16,4 \%$ & $22,2 \%$ & \\
\hline
\end{tabular}

l'Université d'Ottawa. Les données concernant la composition de l'échantillon sont présentées dans le tableau 1.

Comme on le voit, les 39 classes sont réparties inégalement entre les quatre facultés de l'échantillon. Il en est de même de la répartition des étudiants. Il est à noter que l'importance relative de chaque faculté dans l'échantillon ne correspond pas forcément à sa taille au sein de l'Université.

\section{Caractéristiques des personnes interrogées}

Caractéristiques des étudiants. Selon Ellett (1994), Henriksen (1995), Light, Singer et Willett (1990) et Wade (1995), on ne peut s'intéresser à étudier la variation dans l'apprentissage sans, en même temps, se demander, par exemple, les hommes réussissent-ils mieux que les femmes en mathématiques? Les étudiants qui ont obtenu une moyenne plus élevée à l'issue de l'école secondaire sont-ils, dans la même mesure, plus satisfaits de leurs études universitaires que ceux qui étaient moins brillants? Le fait, pour les étudiants du premier cycle, d'appartenir à tel ou tel groupe culturel ou d'avoir un enseignant qui présente telle ou telle caractéristique individuelle, augmente-t-il leurs chances de se rendre aux études supérieures? Ou le fait, pour eux, d'avoir des ambitions ou des projets professionnels a-t-il une influence sur leur engagement face à leurs études (enseignement-apprentissage)? Ce sont ces questions qui, parmi d'autres, ont guidé le choix des caractéristiques qui, directement ou indirectement, influencent soit la perception du climat d'apprentissage, soit la satisfaction des étudiants face à l'enseignement, soit la relation entre ces deux phénomènes. 
Dix caractéristiques des étudiants ont été ainsi retenues et les résultats de la répartition se présentent comme suit: les femmes constituent $61 \%$ de l'échantillon; selon la variable âge, les pourcentages cumulatifs révèlent que $83,3 \%$ des étudiants qui forment l'échantillon ont un âge égal ou inférieur à 24 ans. Par ailleurs, les étudiants de deuxième et troisième années forment la majorité de l'échantillon, soit un total de $69,5 \%$. La première et la quatrième années représentent $30,5 \%$.

Quant au régime d'études, les étudiants à temps plein constituent près de $88 \%$ de l'échantillon, tandis que ceux qui sont inscrits à temps partiel représentent $9 \%$ et la catégorie d'étudiants spéciaux, les 3,1 autres pour cent. Cette répartition paraît près de la réalité qui prévaut en général à l'université.

Les données montrent aussi que des 39 cours dont le climat a été étudié, $4,5 \%$ seulement sont suivis comme cours hors programme, un quart comme cours facultatifs et $70,3 \%$ sont des cours obligatoires dans le programme. Les données portant sur la langue maternelle, quant à elles, indiquent qu'une très forte proportion de $86,3 \%$ des étudiants interrogés sont de langue maternelle française. Les étudiants dont l'anglais est la langue maternelle occupent la dernière place $(5,7 \%)$, devancés par les allophones $(8,0 \%)$. Sans affirmer de manière absolue que ces données reflètent la réalité de l'Université d'Ottawa, nous l'estimons probable sachant que, majoritairement, les étudiants de langue anglaise fréquentent plutôt l'Université Carleton que celle d'Ottawa.

Le lieu où les étudiants ont fait leurs études secondaires correspond "grosso modo" à leur provenance géographique. Bien que la majorité d'entre eux soit originaire de l'Ontario $(57.2 \%)$, une bonne partie des étudiants $(40 \%)$ provient d'une autre province du Canada ou même d'autres pays. En particulier, il ressort que près de $30 \%$ des étudiants ont fait entièrement ou partiellement leurs études secondaires au Québec.

Quant au lieu de logement, 38\% des étudiants de l'échantillon demeuraient chez leurs parents. On peut en déduire que non seulement la majorité des étudiants est originaire de l'Ontario, mais encore elle est issue de la région d'Ottawa-Carleton (c'est-à-dire excluant l'Outaouais québécois). Vingt-neuf pour cent des étudiants préféraient le logement à loyer partagé, tandis que "logement seul" recueille le plus faible pourcentage des cinq types de résidence. De ces trois données on peut présumer que le facteur financier, c'est-à-dire le loyer, est déterminant voire prédominant dans le choix du mode de logement. La résidence sur le campus ne recueille que $9 \%$ dans la faveur des étudiants. 
Enfin, concernant la variable "projet au terme des études", une faible majorité seulement des étudiants interrogés, soit $39 \%$, envisageait de travailler après le premier cycle d'études universitaires. Presque aussi importante, soit $32 \%$, est celle de ceux qui envisageaient de poursuivre leurs études au cycle supérieur. Ceux qui projetaient de poursuivre leurs études tout en travaillant constituent une proportion de $29 \%$.

Caractéristiques des professeurs. Certains enseignants assurant plus d'un cours, l'on comprend que le nombre de professeurs $(\mathrm{N}=34)$ ne corresponde pas à celui de classes de l'échantillon $(\mathrm{N}=39)$. Néanmoins, les résultats de l'étude ne s'en trouvent pas entachés, car, bien que nous ne l'ayons pas vérifié par les instruments utilisés, nous croyons que même si un professeur est titulaire de plusieurs cours, le climat de ceux-ci n'est guère identique.

Quatre caractéristiques des professeurs ont été retenues: le sexe, l'âge, le grade universitaire et l'ancienneté, et les données les concernant se présentent comme suit: l'échantillon est constitué, en majorité, de professeurs de sexe masculin, soit près de $80 \%$. La répartition selon l'âge, quant à elle, montre que $38,3 \%$ des professeurs se situent entre 35 et 45 ans; ceux qui sont âgés de 55 ans et plus ne forment que $16,1 \%$ de l'échantillon. Ceci permet de conclure que ce dernier est constitué de professeurs relativement jeunes, car le pourcentage cumulatif de ceux âgés de moins de 55 ans est élevé $(83,9 \%)$.

En ce qui concerne le grade universitaire, une forte majorité de $77,8 \%$ détient un doctorat; les $13,7 \%$ qui ont une maîtrise étaient généralement, au moment de notre enquête, en instance de thèse de doctorat, tandis qu'il est permis de croire que les $8,5 \%$ "autres" avaient été recrutés en raison de critères spécifiques soit de spécialité connexe à la discipline enseignée, soit d'expérience en enseignement.

Enfin, selon la variable "ancienneté" des professeurs, le plus grand nombre de ces derniers, soit $40,6 \%$, se situe dans la catégorie de quatre à neuf ans d'expérience en enseignement. Une bonne proportion de $36,2 \%$ a tout de même 20 ans et plus: ce qui donne un pourcentage cumulatif de 84,3 de professeurs qui ont une ancienneté d'au moins quatre ans, tandis que peu seulement, soit $15,7 \%$, peuvent être considérés comme nouveaux ou débutant la carrière. 


\section{ANALYSE DES DONNÉES}

Dans cette section, nous présentons les résultats concernant respectivement la perception du climat d'apprentissage des étudiants, leur satisfaction et les relations entre les variables du modèle.

\section{La perception du climat d'apprentissage par les étudiants}

Le tableau 2 ci-dessous expose les résultats d'ensemble de la perception des étudiants sur chacune des échelles du climat de la classe telle que mesuré à l'aide du CUCEI-F .

Il ressort du tableau 2 que le climat d'apprentissage n'est pas perçu de la même manière pour toutes les classes. Cette différenciation apparaît dans l'écart entre ses différentes dimensions. En effet, l'écarttype varie de 2,63 (pour la personnalisation) à 5,13 (pour la motivation). La moyenne des perceptions, quant à elle, varie de 12,04 à 16,25 respectivement pour l'innovation et la participation, traduisant un degré de consensus croissant dans le même sens.

\section{La satisfaction des étudiants}

Les variables utilisées pour décrire le profil des professeurs permettent d'identifier leurs caractéristiques démographiques ou sociologiques. Cependant, elles ne signifient pas, de soi, un enseignement efficace en salle de classe. On ne peut en déduire que peu de chose quant aux stratégies d'enseignement du professeur et à la façon

Tableau 2

Perception du climat d'apprentissage pour l'ensemble des classes $(\mathrm{N}=39)$

$\underline{\text { Variables }}$

Personnalisation

Participation

Cohésion

Motivation

Intérêt

Innovation

Individualisation $\underline{\text { Minimum Maximum Moyenne Écart-type }}$

$\begin{array}{llll}0,00 & 28,00 & 13,77 & 2,63 \\ 0,00 & 28,00 & 16,25 & 2,95 \\ 0,00 & 28,00 & 14,31 & 4,63 \\ 0,00 & 28,00 & 12,97 & 5,13 \\ 0,00 & 28,00 & 13,41 & 3,53 \\ 0,00 & 28,00 & 12,04 & 3,84 \\ 0,00 & 28,00 & 12,38 & 4,66\end{array}$


dont il interagit avec ses étudiants en classe. D'où, pour mesurer la satisfaction de ces derniers, nous nous sommes fondé sur la perception que ceux-ci ont des qualités pédagogiques de leurs professeurs. À cet effet, rappelons que trois énoncés du questionnaire de l'évaluation de l'enseignement et des cours à l'Université d'Ottawa (QÉEC) ont été utilisés, à savoir, l'aptitude du professeur en tant qu'enseignant, son efficacité à communiquer la matière et sa préparation des cours. Les résultats présentés dans le tableau 3 ci-dessous reflètent une variation évidente dans la satisfaction des étudiants.

Les moyennes pour les trois aspects de la satisfaction varient en effet de 2,74 (aptitude) à 3,22 (préparation). L'écart-type varie de 0,95 (pour la préparation des cours) à 1,06 (pour l'efficacité). En d'autres termes, les étudiants jugent ou perçoivent de manière différenciée l'aptitude de leurs professeurs en tant qu'enseignants, leur efficacité à communiquer la matière, et le soin qu'ils mettent à préparer leurs cours. Donc, leur degré de satisfaction varie aussi.

\section{Les relations entre les variables du modèle}

L'analyse porte ici successivement sur les relations 1) entre les caractéristiques des étudiants et leur perception du climat d'apprentissage; 2) entre ces mêmes caractéristiques et la satisfaction; 3) entre les caractéristiques des professeurs et le climat; 4) entre ces mêmes caractéristiques et la satisfaction des étudiants; 5) entre le climat (globalement et chacune de ses échelles prises individuellement) et la satisfaction des étudiants.

Tableau 3

Sommaire de la satisfaction des étudiants $(N=39)$

Aspects de la satisfaction Minimum Maximum Moyennes Écarts-T.

$\begin{array}{lcccc}\text { Aptitude } & 0,00 & 4,00 & 2,74 & 1,05 \\ \text { Efficacité } & 0,00 & 4,00 & 2,86 & 1,06 \\ & & & & \\ \text { Préparation } & 0,00 & 4,00 & 3,22 & 0,95\end{array}$


L'influence des caractéristiques des étudiants sur leur perception du climat de la classe et sur leur satisfaction. Certaines caractéristiques des étudiants, des cours et des professeurs sont mesurées par des variables dichotomiques; c'est le cas du sexe des intervenants (homme $=\mathrm{M} /$ femme $=\mathrm{F}$ ), du régime d'étudiant (temps complet/temps partiel), de la catégorie de cours (obligatoire/facultatif), etc. Dans ces cas, nous avons eu recours au test $\underline{t}$. Les résultats de cette analyse sont présentés au tableau 4 ci-dessous; ce sont seulement les différences les plus élevées qui apparaissent ici. Pour les caractéristiques mesurées sur des échelles de type nominal où plus de deux choix sont possibles, une analyse de la variance a été utilisée. Le tableau 4 présente également les différences significatives de cette analyse, mais en commençant par les plus basses.

Le tableau 4 ci-dessous montre que les étudiants et les étudiantes des quatre années perçoivent le climat de la même manière; la seule différence significative selon la variable 'sexe' réside dans leur satisfaction par rapport à la préparation des cours, les femmes en étant plus satisfaites que les hommes.

Selon le régime d'étudiant, les étudiants à temps plein et à temps partiel ne perçoivent différemment de façon significative que la composante "motivation" du climat et leur satisfaction par rapport à la qualité pédagogique varie de manière significative pour l'aptitude à enseigner et la préparation de cours des professeurs.

La catégorie de cours, soit obligatoire et facultatif, n'influence pas la satisfaction des étudiants par rapport à l'enseignement telle que mesurée à l'aide du QÉEC. En revanche, la catégorie de cours contribue de manière significative à la variation dans la perception de toutes les composantes du climat, excepté la personnalisation.

Le tableau 4 montre aussi une différence significative dans la perception du climat d'apprentissage et dans la satisfaction des étudiants selon la langue maternelle: le français ou une autre. Elle porte notamment sur quatre composantes du climat (la personnalisation, la participation, la motivation et l'innovation) ainsi que sur un aspect de l'enseignement (l'efficacité).

Selon le lieu des études secondaires, les aspects personnalisation et intérêt pour la tâche sont perçus différemment, tandis qu'il n'y a pas de différence significative dans la satisfaction. 
Tableau 4

Synthèse des différences significatives dans la variation de la perception du climat d'apprentissage et de la satisfaction des étudiants, par rapport à leurs caractéristiques $(\mathrm{N}=984)$, suivant le test $\underline{t}$

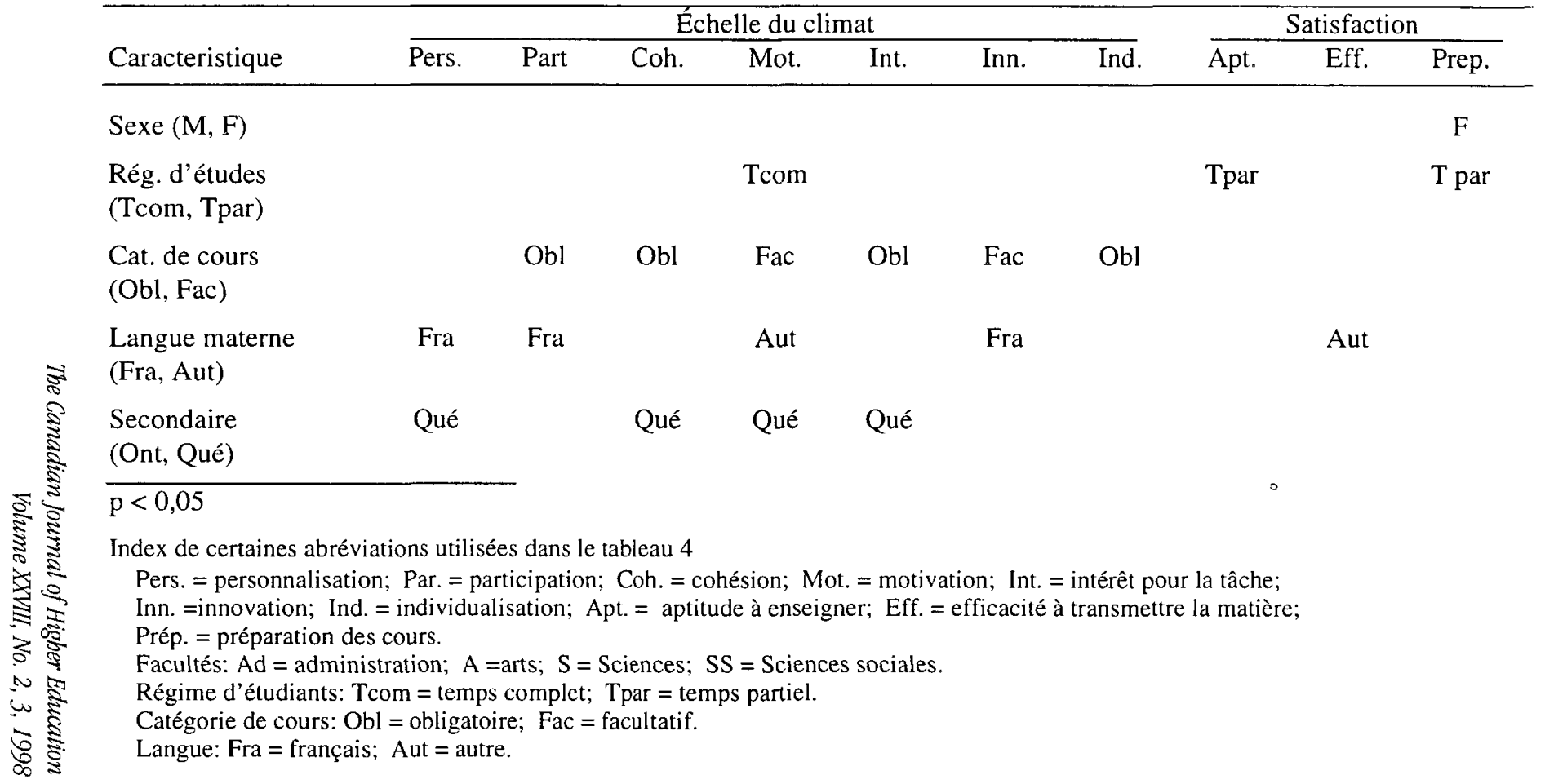




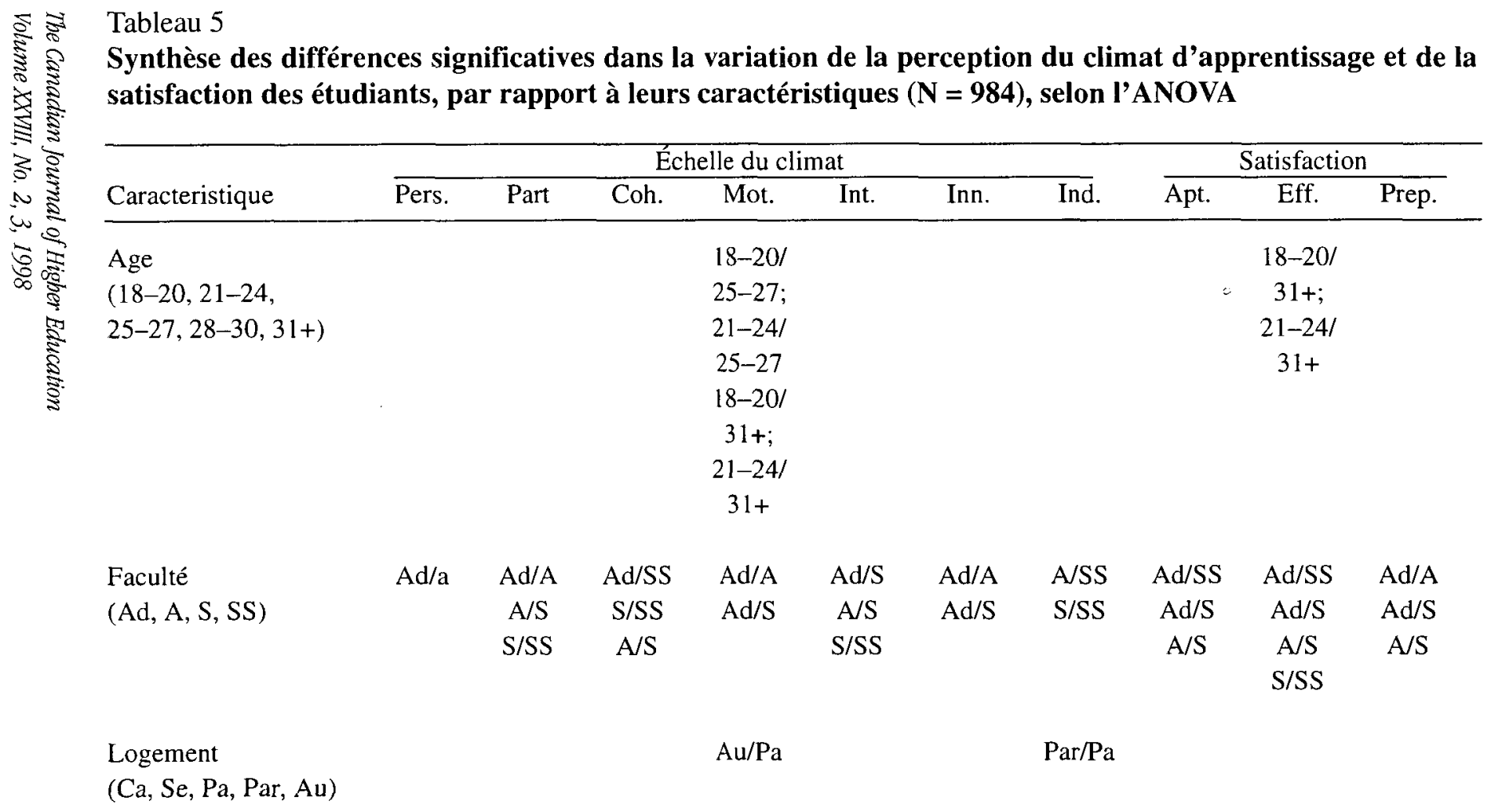


Tableau 5 (cont.)

\begin{tabular}{|c|c|c|c|c|c|c|c|c|c|c|}
\hline \multirow[b]{2}{*}{ Caracteristique } & \multicolumn{7}{|c|}{ Échelle du climat } & \multicolumn{3}{|c|}{ Satisfaction } \\
\hline & Pers. & Part & Coh. & Mot. & Int. & Inn. & Ind. & Apt. & Eff. & Prep. \\
\hline $\begin{array}{l}\text { Projets } \\
\text { (Tr, Ét, Ét+Tr) }\end{array}$ & & & & $\begin{array}{c}\mathrm{E} E+\mathrm{Tr} \\
\mathrm{Tr} \\
\mathrm{E} \mathrm{E}+\mathrm{Tr}\end{array}$ & & $\begin{array}{c}\text { Ét+Tr/ } \\
\text { Ét } \\
\text { Tr/Ét }\end{array}$ & $\begin{array}{c}\text { Ét+Tr/ } \\
\text { Ét }\end{array}$ & $\begin{array}{c}\text { Ét+Tr/ } \\
\text { Ét } \\
\text { Tr/Ét }\end{array}$ & $\begin{array}{c}\text { Tr/Ét } \\
\text { Ét+Tr/ } \\
\text { Ét }\end{array}$ & \\
\hline
\end{tabular}

$\mathrm{p}<0,05$

Index de certaines abréviations utilisées dans le tableau 4

Pers. = personnalisation; Par. = participation; Coh. = cohésion; Mot. = motivation; Int. = intérêt pour la tâche;

Inn. =i nnovation; Ind. = individualisation; Apt. = aptitude à enseigner; Eff. = efficacité à transmettre la matière;

Prép. = préparation des cours.

Facultés: $\mathrm{Ad}=$ administration; $\mathrm{A}=$ arts; $\mathrm{S}=$ Sciences; $\mathrm{SS}=$ Sciences sociales.

Logement: $\mathrm{Ca}=$ sur le campus; $\mathrm{Se}=$ seul; $\mathrm{Par}=$ chez les parents; $\mathrm{Pa}=$ logement partagé.

Projets au terme des études: $\operatorname{Tr}=$ travail; Ét = études supérieures. 
Si l'on considère la variable âge, il apparaît au tableau 5 ci-dessus que les étudiants perçoivent différemment la composante "motivation" du climat d'apprentissage, ainsi que l'aspect "efficacité" de l'enseignement. Les différences significatives, quant à la motivation, existent notamment entre les perceptions des tranches d'âge de 18 à 20 ans et 25 à 27 ans ainsi que de 21 à 24 et 25 à 27 , d'une part, et entre celles de 18 à 20 ans et 31 ans et plus ainsi qu'entre celles de 21 à 24 et de 31 et plus, d'autre part. Celles constatées dans la satisfaction existent entre les opinions des étudiants âgés de 18 à 20 ans et de 31 ans et plus ainsi qu'entre celles de ceux âgés de 21 à 24 et 31 et plus. En effet, il apparaît que les étudiants plus jeunes (18 à 24 ans) perçoivent moins favorablement la variable "motivation" du climat d'apprentissage et leur satisfaction par rapport à l'efficacité de l'enseignement est moins élevée que celle des étudiants plus âgés (31 ans et plus).

La faculté à l'intérieur de laquelle s'offre le cours, ou en fait la discipline enseignée, paraît la caractéristique qui constitue la source la plus forte de variation tant dans la perception du climat que dans la satisfaction des étudiants. En effet, la perception de toutes les composantes de celui-là et de tous les aspects de celle-ci varie de manière significative d'une faculté à l'autre. Le tableau 5 indique en particulier que c'est dans la satisfaction des étudiants par rapport à l'efficacité des professeurs que la variation est la plus significative entre les facultés, tandis que la perception de la variable "personnalisation" du climat varie de manière significative seulement entre la faculté d'administration et celle des arts.

Par rapport au lieu de logement les différences significatives ne sont constatées que dans la perception de deux composantes du climat, notamment la motivation et l'innovation, tandis qu'il n'y en a aucune pour les trois aspects de la satisfaction des étudiants. La variation de perception pour la motivation se situe entre le type de "autre" et "logement partagé". Pour la variable "innovation" la différence de perception significative existe seulement entre les opinions des étudiants qui logent chez leurs parents et ceux qui partagent le logement avec une autre personne.

Enfin, selon leurs projets immédiats au terme des études, d'une part, les étudiants perçoivent différemment les trois composantes du climat suivantes: la motivation, l'innovation et l'individualisation, et, d'autre part, leur satisfaction par rapport à l'enseignement varie pour 
l'aptitude et l'efficacité des professeurs. Les différences sont significatives particulièrement dans la perception des étudiants qui projettent de combiner études et travail et ceux qui choisissent seulement de poursuivre leurs études.

En somme, dans cette analyse des relations au niveau micro ou individuel, nous avons utilisé un grand nombre de sujets $(\mathrm{N}=984)$ dont les caractéristiques ont été associées avec les perceptions sur le climat d'apprentissage et la satisfaction. Un tel échantillon a contribué à rendre l'analyse plus fiable. En revanche, certains professeurs assurant plus d'un cours dont le climat a été étudié, les données au niveau des classes (tableau 6) ont forcément été répétitives. L'erreur d'estimation sera donc plus grande ici que dans l'analyse faite au niveau individuel.

\section{L'INFLUENCE DES CARACTÉRISTIQUES DES PROFESSEURS SUR LA PERCEPTION DU CLIMAT ET SUR LA SATISFACTION DES ÉTUDIANTS}

Comme pour les caractéristiques des étudiants, seules les différences significatives $(p<0,05)$ entre les variables obtenues par le test $\underline{t}$ et l'analyse de la variance, sont exposées. L'analyse explore notamment l'incidence des caractéristiques des professeurs sur la variation dans la perception du climat d'apprentissage et de la satisfaction des étudiants. Les résultats sont présentés dans le tableau 6 ci-dessous de la même manière que dans les tableaux 4 et 5 précédents. Cependant, étant donné que, dans la présente analyse, le test $\underline{t}$ n'a porté que sur une seule variable, à savoir le sexe, les résultats concernant cette dernière sont présentés, sans risque de confusion, dans le même tableau (6) que ceux de l'analyse de la variance.

Il apparaît que les étudiants des classes dont les professeurs sont des femmes perçoivent plus positivement deux composantes du climat, à savoir la personnalisation et la motivation, et sont plus satisfaits de la préparation des cours que ceux des classes dont les enseignants sont des hommes.

Par ailleurs, c'est en regard de la caractéristique "âge" des professeurs qu'il existe plus de variation dans la perception du climat d'apprentissage et dans la satisfaction par rapport à l'enseignement. En effet, il ressort que les étudiants perçoivent plus favorablement la personnalisation, l'innovation ainsi que l'individualisation, et ils sont 
Tableau 6

Synthèse des différences significatives dans la variation de la perception du climat d'apprentissage et de la satisfaction des étudiants, par rapport aux caractéristiques des professeurs $(N=39$

\begin{tabular}{|c|c|c|c|c|c|c|c|c|}
\hline \multirow[b]{2}{*}{ Caracteristique } & \multicolumn{7}{|c|}{ Échelle du climat } & \\
\hline & Pers. & Part & Coh. & Mot. & Int. & Inn. & Ind. & Apt. \\
\hline $\operatorname{Sexe}(M, F)$ & $\mathrm{F}$ & & & $\mathrm{F}$ & & & & \\
\hline Age & $25-34 / 55+$ & & & & & $45-54 / 55$ & $45-54 / 55$ & $25-34 / 55+$ \\
\hline $\begin{array}{l}(25-34,35-44, \\
45-54,55+)\end{array}$ & $\begin{array}{l}35-44 / 55+ \\
45-54 / 55+\end{array}$ & & & & & & & $\begin{array}{l}35-44 / 55+ \\
45-54 / 55+\end{array}$ \\
\hline
\end{tabular}

Grade

Aut/D

(Ma, D, Aut)

Ancienneté

$4-9 / 20+$

$(1-3,4-9$,

$10-19,20+)$

$\mathrm{p}<0,05$

Pers. $=$ personnalisation; Par.$=$ participation; Coh. $=$ cohésion; Mot. $=$ motivation; Int. $=$ intérêt pour la tâche;

Inn. =i nnovation; Ind. = individualisation; Apt. = aptitude à enseigner; Eff. = efficacité à transmettre la matière;

Prép. $=$ préparation des cours.

$\mathrm{Ma}=$ maîtrise $; \mathrm{D}=$ doctorat; Aut $=$ Autre $\mathbf{M}=$ homme;

$\mathrm{F}=$ femme; Pers. $=$ personnalisation; Par. = participation; Coh. $=$ cohésion; 
plus satisfaits de l'aptitude pédagogique dans les classes dont les professeurs sont plus âgés (55 ans et plus) que de ceux qui sont plus jeunes ( 25 à 54 ans).

Le grade universitaire et l'ancienneté des professeurs, enfin, n'ont d'influence significative que sur la perception de deux composantes du climat, soit respectivement la motivation et la personnalisation. En effet, selon la perception des étudiants, les professeurs détenteurs d'un doctorat motivent plus les étudiants que ceux qui possèdent un "autre" grade universitaire (que la maîtrise). Quant à la différence de perception de la variable "personnalisation" liée à l'ancienneté, le test de Scheffé précise qu'elle existe entre les classes dont les professeurs ont une expérience de 4 à 9 ans et 20 ans et plus. En d'autres termes, les étudiants perçoivent plus positivement la dimension "personnalisation" du climat d'apprentissage des classes dont les professeurs sont plus expérimentés en enseignement.

Une conclusion se dégage des analyses qui viennent d'être faites: toutes les variables intrants retenues dans la recherche, c'est-à-dire les caractéristiques individuelles des étudiants et des professeurs, excepté l'année d'étude, ont quelqu'influence sur la perception du climat et la satisfaction des étudiants.

\section{L'influence du climat de la classe global et par échelle sur la satisfaction des étudiants}

Nous voulons, ici, tenter de répondre à la question de recherche suivante: quelle est la part du climat global et de chacune de ses échelles dans l'explication des variations dans la satisfaction des étudiants? Il s'agit, en fait, de déterminer quel pourcentage de la variation de cette dernière s'explique par le premier. L'analyse de régression linéaire a été utilisée à cet effet. À partir des paramètres d'estimation, les équations de régression sont formulées et présentées dans le tableau 7 ci-dessous.

Globalement, en effet, le climat d'apprentissage $\left(r^{2}\right)$ explique $35 \%$ de la variation dans la satisfaction des étudiants par rapport à l'aptitude à enseigner des professeurs, $33 \%$ en ce qui concerne l'efficacité à transmettre la matière, et $17 \%$ quant à la préparation des cours. Quelques composantes ou échelles individuelles du climat tel que mesuré par le CUCEI-F paraissent avoir une influence déterminante dans cette variation. Il s'agit notamment, en ordre de pourcentage décroissant (coefficient de corrélation), des variables motivation, participation, personnalisation, intérêt pour la tâche et innovation. Ces variables 
Tableau 7

Coefficients de corrélation des trois aspects de la satisfaction avec les sept échelles du climat $(\mathrm{N}=929)$

\begin{tabular}{|c|c|c|c|}
\hline \multirow[t]{2}{*}{$\underline{\text { Climat }}$} & \multicolumn{3}{|c|}{ Satisfaction } \\
\hline & Apt. & Eff. & Prép. \\
\hline \multicolumn{4}{|l|}{ Constante } \\
\hline Coef. de corr. & 1,15 & 1,05 & 2,24 \\
\hline Probabilité & $0,0001^{*}$ & $0,0001 *$ & $0,0001^{*}$ \\
\hline \multicolumn{4}{|l|}{ Personnalisation } \\
\hline Coef. de corr. & 0,24 & 0,14 & 0,28 \\
\hline Probabilité & 0,003 & 0,08 & 0,0005 \\
\hline \multicolumn{4}{|l|}{ Participation } \\
\hline Coef. de corr. & $-0,29$ & $-0,22$ & $-0,22$ \\
\hline Probabilité & $0,0001 *$ & 0,002 & 0,0015 \\
\hline \multicolumn{4}{|l|}{ Cohésion } \\
\hline Coef. de corr. & $-0,07$ & $-0,01$ & $-0,06$ \\
\hline Probabilité & 0,08 & 0,79 & 0,15 \\
\hline \multicolumn{4}{|l|}{ Motivation } \\
\hline Coef. de corr. & 0,46 & 0,55 & 0,35 \\
\hline Probabilité & $0,0001^{*}$ & $0,0001^{*}$ & 0,03 \\
\hline \multicolumn{4}{|l|}{ Intérêt pr tâche } \\
\hline Coef. de corr. & 0,17 & 0,32 & 0,13 \\
\hline Probabilité & 0,004 & $0,0001 *$ & 0,03 \\
\hline \multicolumn{4}{|l|}{ Innovation } \\
\hline Coef. de corr. & 0,26 & 0,13 & 0,06 \\
\hline Probabilité & 0,0002 & 0,07 & 0,35 \\
\hline \multicolumn{4}{|l|}{ Individualis. } \\
\hline Coef. de corr. & 0,17 & 0,10 & 0,01 \\
\hline Probabilité & 0,001 & 0,06 & 0,77 \\
\hline $\mathrm{r}^{2}$ maximum & $0,35^{* *}$ & 0,33 & 0,17 \\
\hline Probabilité (alpha) & $0,0001 *$ & $0,0001 *$ & $0,0001 *$ \\
\hline
\end{tabular}

\footnotetext{
$* p \geq 0,05$

$* * \mathrm{p} \geq 0,01$
} 
expliquent la majeure partie de la variation de la satisfaction des étudiants telle que nous l'avons mesurée. La cohésion et l'individualisation ont une faible influence.

\section{DISCUSSION}

Le premier objectif de notre recherche était d'explorer l'existence et la nature de la relation entre le climat d'apprentissage et la satisfaction des étudiants à l'Université d'Ottawa. L'étude confirme, de fait, l'existence d'une certaine relation - sinon d'une relation certaine - entre le climat d'apprentissage et la satisfaction des étudiants. Cette relation est, selon notre modèle conceptuel, directe et indirecte, voire réciproque. Quoique nous n'ayons pas étudié ce dernier aspect, nous souscrivons, par présomption, à la conclusion en ce sens d'Ellett (1994), d'EvansHarvey (1995), de Murray (1980) et de Walberg (1969).

Il importe de préciser cependant que ce type de liaison n'est pas une relation de causalité ou de "cause à effet". Elle est plutôt une sorte d'interdépendance de deux variables en présence d'autres facteurs: c'est une relation fonctionnelle d'influence. En effet, le climat d'apprentissage "explique", c'est-à-dire permet de comprendre, ou "influence" la variation dans la satisfaction des étudiants, soit directement, soit en présence d'autres facteurs, telles que les caractéristiques de ces derniers, des professeurs et des environnements interne et externe de l'université.

La conclusion de notre étude quant à la relation du climat global de la classe et la satisfaction concorde, elle aussi, avec d'autres études antérieures (Anderson, Ryan \& Shapiro, 1989; Brookover et al., 1979; Corno \& Snow, 1986; Dippelhofer-Steim, 1986; Donnelly, 1982; Holzemer, 1975; Ramsden, 1986).

Le second objectif de notre recherche était de comparer, d'une part, les climats d'apprentissage, et d'autre part, leur influence sur la satisfaction des étudiants de différentes classes. Les résultats à ce sujet ont permis de constater que le climat de la classe ainsi que son influence sur la satisfaction varient. Mais la comparaison de cette variation entre différentes classes s'est avérée d'autant plus difficile que les cours dont le climat a été étudié sont hétérogènes. Il a été néanmoins possible de le réaliser par rapport aux caractéristiques internes des classes: celles des étudiants et des professeurs.

Ainsi, contrairement à Donnelly (1982) et Holzemer (1975), nous avons trouvé une relation significative entre le sexe des étudiants et leur 
perception du climat d'apprentissage ainsi qu'avec leur satisfaction par rapport à l'aspect "efficacité de l'enseignement"; nos résultats concordent néanmoins avec la conclusion d'Astin (1977), d'Ellett (1994) et de Tidball (1973).

Par ailleurs, selon Donnelly (1982) et Holzemer (1975), les étudiants des années avancées semblent plus généreux dans leur évaluation de l'enseignement que ceux des années inférieures, tandis que d'après notre étude, l'année d'étude n'a de relation significative ni avec le climat de la classe ni avec la satisfaction des étudiants par rapport à l'enseignement et au cours. Une étude commandée par le Comité du Sénat pour l'évaluation de l'enseignement et des cours de l'Université d'Ottawa (1992) utilisant, comme nous, le QÉEC avec un échantillon formé de classes des départements de psychologie, sociologie, économie et criminologie conclut que la catégorie de cours (obligatoire-facultatif) n'influence nullement de façon significative la satisfaction des étudiants. Donnelly (1982) et Holzemer (1975) l'attestent également.

Le pourcentage de la variation de la satisfaction des étudiants qu'explique le climat d'apprentissage paraît d'autant plus appréciable que la majeure partie du temps, des activités éducatives et du développement des étudiants à l'université se passent sur le campus en dehors de la classe. En outre, la satisfaction des étudiants en relation de laquelle l'influence du climat de la classe a été examinée dans cette recherche, n'est qu'un indicateur parmi plusieurs autres de l'apprentissage, celui-ci étant considéré comme un processus dynamique et complexe. C'est précisément cette complexité et cette richesse tant du phénomène de climat que de celui d'apprentissage que notre modèle conceptuel a le mérite de montrer: le processus d'enseignementapprentissage n'est pas un acte mécanique ou purement bureaucratique; il est un acte humain et un lieu de rôles, d'interrelations et d'émotions souvent difficiles à mesurer. On doit donc se garder de considérer a priori l'influence du climat d'apprentissage comme négligeable.

Nous croyons d'ailleurs qu'un climat d'apprentissage absolu et idéal n'existe pas; il est contextuel, pourvu que les étudiants s'y développent, s'y sentent bien et que l'écart entre ses composantes soit réduit. C'est ce que nous appelons une importance existentielle - plutôt que simplement statistique - de la relation entre le climat d'apprentissage et la satisfaction des étudiants. C'est cette importance existentielle que théoriciens et praticiens de l'éducation doivent s'attacher à comprendre 
et à privilégier dans la recherche des politiques de l'amélioration du processus d'enseignement-apprentissage.

Il existe plusieurs façons de procéder pour "comprendre" celui-ci. L'approche relationnelle ou systémique nous est apparue mieux appropriée et a été privilégiée dans notre étude. L'enseignementapprentissage y est appréhendé comme un tout, un processus dynamique mais complexe où interagissent étudiants et professeurs avec leurs caractéristiques individuelles, leurs expériences et méthodes personnelles d'apprenants et d'enseignants, les caractéristiques organisationnelles de l'université et les perceptions qu'ils en ont, ainsi que la discipline ou matière enseignée et l'environnement externe. Dans cette perspective, la classe est considérée comme un sous-système social de l'université, ayant sa sous-culture et son propre climat constitué de caractéristiques formelles et informelles que les étudiants perçoivent dans la lorgnette de leurs caractéristiques individuelles et de leurs besoins.

\section{LIMITES DE LA PRÉSENTE RECHERCHE}

Cette recherche comporte certaines limites dues soit au choix délibéré du sujet de l'étude, soit à sa complexité, soit aux contraintes de temps et d'autres ressources. Ainsi, elle n'est pas une étude longitudinale et n'utilise pas les perceptions des professeurs ni la forme "idéale" de l'instrument du climat de la classe; celui-ci ne mesure d'ailleurs qu'un nombre restreint de caractéristiques ou aspects du climat de la classe à l'université — tout instrument ne sera toujours qu'un échantillon de dimensions du phénomène à mesurer -; la comparaison des climats est faite seulement en fonction des caractéristiques des étudiants et des professeurs retenues et non des disciplines, et elle porte uniquement sur les données subjectives recueillies auprès des étudiants, sans les fonder sur une observation directe ni les confronter aux données objectives du système universitaire entier.

\section{CONCLUSION}

Notre recherche n'a touché qu'à un infime - mais complexe phénomène de l'organisation universitaire: le climat de la classe. Son influence a été analysée sur un indicateur du processus d'apprentissage tout aussi complexe. Notre méthodologie de la recherche a privilégié les perceptions des étudiants et tenté de comprendre comment ces derniers 
vivaient et interprétaient leur contexte d'apprentissage. Cette vision subjective d'un sous-système de l'université a des limites, certes; néanmoins elle n'est ni vraie ni fausse et aucun jugement global ne peut en être tiré. Elle est simplement celle des étudiants et guide leur action. Elle s'enracine dans leur espace de travail, de contraintes et de rapports entre eux et avec les enseignants. Elle se fonde aussi sur leurs besoins et leurs projets professionnels et sociaux.

Il nous est apparu qu'il existe une relation certaine entre cette perception du climat de la classe et la satisfaction "scolaire" des étudiants. Celui-là n'est certes pas une panacée ni le seul facteur qui explique la variation de celle-ci, mais il en fait la différence. Chercheurs, pédagogues et administrateurs de l'éducation se doivent donc de le prendre en compte. Mais sa variation et sa complexité que la présente étude a également mises en lumière ne sont pas sans poser des problèmes de compréhension, de mesure et de gestion.

Les résultats des travaux antérieurs s'étaient révélés inconstants: des facteurs qui, selon certaines études, paraissent déterminants n'ont pas forcément, selon d'autres, d'influence déterminante sur l'apprentissage. C'est à cette même conclusion générale que nous arrivons au terme de notre enquête. Le débat sur les variations dans l'apprentissage des étudiants n'est donc pas clos.

Nous suggérons quelques pistes pour la recherche qui doit s'intensifier particulièrement en langue française sur la classe à l'université: les études ultérieures, de préférence longitudinales, devraient inclure non seulement les opinions des étudiants sur le climat idéal ou souhaité, mais aussi les perceptions des professeurs sur le climat de la classe et utiliser des techniques d'analyse plus poussées; l'utilisation d'un instrument de mesure complémentaire, par exemple celui de type d'observation directe, pourrait assurer un surcroît d'efficacité et de validité; les études ultérieures devraient également utiliser un plus grand échantillon et retenir un plus grand nombre de variables reflétant davantage la réalité universitaire et socio-culturelle. 


\section{Références}

Aitken, N. (1982). College student performance, satisfaction and retention. Journal of Higher Education, 53(1), 32-50.

Anderson, C.S. (1982). The search for school climate: A review of the research. Review of Educational Research, 52(3), 368-420.

Anderson, L.W., Ryan, D.W., \& Shapiro, B.J. (1989). The IEA classroom environment study. Toronto, ON: Pergamon Press.

Astin, A.W. (1977). Four critical years. San Francisco, CA: Jossey-Bass.

Brookover, W.B., Beady, C., Flood, P., Scheitzer, J., \& Weisenbaker, J. (1979). School social systems and student achievement. New York, NY: Praeger.

Brunet, L. (1987). Le climat organisationnel et le milieu scolaire. In C. Barnabé, \& H.C. Girard, Administration scolaire: Théorie et pratique. Chicoutimi, PQ: Gaëtan Morin.

Carothers, R.L.. \& Sevingy, M.-L. (1993). Classism and quality. New Directions for Institutional Research, 78, 13-15.

Coleman, J.S., Campbell, E.Q., Hobson, C.J., McPartland, J., Mood, A.M., Weinfeld, F.D., \& York, R.L. (1966). Equality of educational opportunity. Washington: US Printing Office.

Comité (Le) du Sénat pour l'évaluation de l'enseignement et des cours de l'Université d'Ottawa (1992). Évaluation de l'enseignement. Renseignements généraux à l'intention des étudiants et étudiantes.

Corno, L., \& Snow, R.E. (1986). Adapting teaching to individual differences among learners. In M. Wittrock (Éd.), Handbook of research on teaching. New York, NY: MacMillan.

Dippelhofer-Steim, B. (1986). How to measure university environment? Methodological implications and some empirical findings. Higher Education, 15(5), 475-495.

Donnelly, B. (1982). The relationship between school management patterns and environmental press on students in grade seven and eight. Thèse de doctorat, Université d'Ottawa.

Ellett, D.-R., (1994). Improving academic achievement of eleventh grade basic U.S. history students through the sse of cooperative learning. Master's Project. Saint Xavier University, Illinois.

Evans-Harvey, C. (1995). Creating a positive climate for learning in higher education. Canadian Society for the Study of Higher Education. Professional File, 13.

Fourgous, J.-M., \& Iturralde, B. (1991). Mesurer et améliorer le climat social dans l'entreprise. Paris: Les éd. d'Organisation.

Fraser, B.J., Treagust, D.F., Williamson, J.C., \& Tobin, K.G. (1986). Validation and application of the College and University Classroom Environment Inventory (CUCEI). In B.J. Fraser, The Study of Learning Environments, Vol. 2. Perth: Curtin University of Technology. 
Fraser, B.J., \& Rentoul, A.J. (1982). Relationship between school level and classroom level environment. The Alberta Journal of Educational Research, 28(3), 212-225.

Gartner, W.-B. (1993). Dr. Deming comes to class. Journal of Management Education, 17(2), 143-158.

Genn, J.M. (1984). Research into the climate of Australian schools, colleges and universities: Contribution and potential of need-press theory. Australian Journal of Education, 28, 227-248.

Hanushek, E. (1972). On the value of equality of educational opportunity as a guide to public policy. In Mosteller \& Maynihan (Éd.), On equality of educational opportunity. New York, NY: Random House.

Henriksen, J.-A.-S. (1995). The influence of race and ethnicity on access to postsecondary education and the college experience. ERIC Digest. Washington, DC: Office of Educational Research and Improvement.

Holzemer, W.L. (1975). Student ratings of instructional effectiveness and their relationship to the college classroom environment. Thèse de doctorat, Syracuse University.

Hoy, W.K., \& Forsyth, P.B. (1986). Effective supervision: Theory into practice. New York, NY: Randow House.

Light, R.J., Singer, J.D., \& Willett, J.B. (1990). By design: Planning research on higher education. Cambridge, MA: Harvard University Press.

Marcelo, C. (1988). Research on psychosocial environment evaluation at university classroom: C.U.C.E.I. to the Spanish Educational Context. Paper presented at the annual meeting of the American Educational Research Association, New Orleans, LA.

Michaud, P., Forgette-Giroux, R., \& Richard, M. (1989). Le climat d'apprentissage: Analyse conceptuelle. The Journal of Educational Thought/Revue de la Pensée Éducative, 23(1), 27-43.

Moos, R.H. (1979). Evaluating educational environment. San Francisco, CA: Jossey-Bass.

Murray, H.G. (1980). Evaluating university teaching: A review of research. Toronto, ON: Ontario Confederation of University Faculty Associations.

Pace, C.R. (1961). Diversity of college environments. Journal of the National Association of Women Deans and Counsellors, 25(1), 21-26.

Ramsden, P. (1986). Students and quality. In G.C. Moodie, Standards and criteria in higher education. Guildford: SRHE \& NFER-Nelson.

Savoie, A. (1987). Le perfectionnement des ressources humaines en organisation. Théories, méthodes et applications. Montréal, PQ: Les éd. Agence d'ARC Inc.

Serey, T.-T. (1993). When things go wrong: Using feedback to nanage the classroom learning climate. Journal of Management Education, 17(2), 243-252. 
Tagiuri, R. (1968). The concept of organizational climate. In R. Tagiuri, \& G.H. Litwin (Éd.), Organizational climate: Exploration of a concept. Boston, MA: Harvard University, Division of Research, Graduate School of Business Administration.

Tidball, M.E. (1973). Perspective on academic women and affirmative action. Education Record, 54, 130-135.

Wade, R.C. (1995). Teacher education. Students' view on class discussion: implication for fostering critical reflection. Teaching and Teacher Education, 10(2), 231-243.

Walberg, H.J. (1969). Social environment as a mediator of classroom learning. Journal of Educational Psychology, 60, 443-448.

\section{Annexe}

Questionnaire de cueillette des données sur les caractéristiques des étudiants, le climat psychosocial d'apprentissage au collège et à l'université (CUCEI-F) et la satisfaction des étudiants par rapport à l'enseignement (QÉEC)

\section{Directives aux étudiants}

Le but de ce questionnaire est d'obtenir votre opinion du cours que vous suivez en ce moment. Le questionnaire décrit des situations qui peuvent se présenter dans un cours. J'aimerais vous demander de me donner votre perception de votre cours, c'est-à-dire d'indiquer si les énoncés du présent questionnaire correspondent à ce qui, selon vous, se passe dans votre cours, entre le professeur et les étudiants et entre les étudiants eux-mêmes.

Il n'y a pas de bonnes ou de mauvaises réponses; vous devez simplement indiquer votre opinion sur la situation vécue dans votre cours, en exprimant votre accord ou votre désaccord avec la description qui en est faite dans chaque énoncé.

Les réponses doivent être données sur les feuilles-réponses de l'Université. . ., en noircissant, au crayon, la case surmontée du chiffre (0-4) correspondant à votre opinion. La réponse aux questions non pertinentes ou pour lesquelles vous n'avez pas d'opinion, équivaut à l'opinion 0 . 
Il m'est important d'obtenir vos réponses à toutes les questions. Vous êtes toutefois tout à fait libre de participer ou de ne pas participer, et d'arrêter votre participation en tout temps.

Les réponses sont totalement confidentielles et anonymes, et les données obtenues ne seront pas utilisées dans une autre étude. Le questionnaire est rédigé en utilisant le genre masculin non marqué ou extensif, c'est-à-dire que le masculin réfère alors à tout être, indépendamment de son sexe, et réfère dès lors à un homme, comme à une femme.

\section{Variables démographiques}

1 Sexe: $\quad$ O $\quad M \quad 1 \quad F$

2 Age: $\quad \begin{array}{llllllllll}\mathbf{0} & 18-20 & \mathbf{1} & 21-24 & \mathbf{2} & 25-27 & \mathbf{3} & 28-30 & \mathbf{4} & 31+\end{array}$

3 Années d'étude: $\quad 0$ lre année 1 2e année 23 e année

3 4e année

4 Type d'étudiant: $\quad \mathbf{0}$ temps complet 1 temps partiel

2 étudiant spécial 3 auditeur

5 Faculté qui offre le cours: $\quad 0$ administration $\quad \mathbf{1}$ arts $\quad \mathbf{2}$ sciences

3 sciences sociales

6 Catégorie de cours: Dans votre programme ce cours est ...

0 obligatoire 1 facultatif 2 hors programme

7 Langue maternelle: $\mathbf{0}$ français $\mathbf{1}$ anglais $\mathbf{2}$ autre

8 Lieu où vous avez complété vos études secondaires:

0 Ontario 1 Québec 2 Ontario et Québec

3 Autres provinces du Canada 4 Autres pays

9 Lieu de logement: au cours de l'année scolaire où demeurez-vous?

0 sur le campus en résidence pour étudiants

1 dans un logement seul

2 dans un logement partagé avec un ou plusieurs autres étudiants

3 chez vos parents

4 autre

10 Projet au terme des études: après vos études souhaitez-vous

0 aller sur le marché de travail

1 poursuivre les études de cycle supérieur

2 poursuivre les études de cycle supérieur tout en travaillant?

\section{Variables relatives à la perception du climat}

Répondez à toutes les questions, même si certaines se ressemblent.

0 tout à fait en désaccord 1 plutôt en désaccord

2 plus ou moins d'accord $\mathbf{3}$ plutôt d'accord $\mathbf{4}$ tout à fait d'accord 
11 Le professeur tient compte des besoins des étudiants.

12 Le professeur préfère parler plutôt qu'écouter.

13 Cette classe est composée d'étudiants qui se connaissent peu.

14 J'ai hâte de venir à ce cours.

15 Je sais exactement ce que je dois faire dans ce cours.

16 Je fais un effort pour bien travailler dans ce cours.

17 Les étudiants de ce cours se connaissent bien.

18 Dans ce cours, des méthodes pédagogiques nouvelles sont rarement utilisées.

19 On permet généralement aux étudiants de travailler à leur propre rythme.

20 Le professeur fait tout son possible pour aider les étudiants.

21 Les étudiants surveillent l'heure: ils ont hâte à la fin du cours.

22 À la fin du cours j'éprouve un sentiment de satisfaction.

23 Dans ce cours, le groupe a souvent tendance à dévier du sujet.

24 Le professeur prépare des activités innovatrices pour les étudiants.

25 Les étudiants ont leur mot à dire dans l'organisation du cours.

26 Le professeur aide tout étudiant qui éprouve des difficultés dans ses travaux.

27 Les étudiants de ce cours ont peu d'occasions de se connaître.

28 C'est un cours qui est mal organisé.

29 Les méthodes d'enseignement de ce cours sont caractérisées par l'innovation.

30 Les étudiants peuvent choisir leurs activités ainsi que la façon de les réaliser.

31 Les étudiants présentent rarement leurs travaux devant le groupe.

32 Ça prend beaucoup de temps pour connaître les autres étudiants de ce cours.

33 Ce cours est ennuyant.

34 Les travaux exigés dans ce cours sont précis, de sorte que je sais ce que j'ai à faire.

35 Les approches pédagogiques permettent aux étudiants de progresser à leur propre rythme.

36 Le professeur ne s'intéresse guère aux problèmes des étudiants.

37 Dans ce cours, les étudiants ont souvent l'occasion d'exprimer leurs opinions.

38 Les étudiants de ce cours apprennent à bien se connaître.

39 J'aime venir à ce cours.

$40 \mathrm{Ce}$ cours commence rarement à temps.

41 Le professeur prépare souvent des activités originales pour ce cours.

42 Le professeur est froid à l'égard des étudiants.

43 Le professeur monopolise les discussions au cours. 
44 Les étudiants de ce cours sont peu intéressés à se connaître.

45 Ce cours est intéressant.

46 Les activités d'apprentissage de ce cours sont claires.

47 Les étudiants ont l'impression de faire le même genre d'activités à chaque cours.

48 C'est le professeur qui décide de ce qui se fera dans ce cours.

49 Le professeur ignore les besoins des étudiants.

50 Les étudiants de ce cours ignorent qui sont les autres étudiants.

51 Ce cours est d'un grand intérêt pour mon choix de carrière.

52 Le style d'enseignement du professeur favorise la créativité des étudiants.

53 Le professeur encourage les étudiants à s'intéresser à l'organisation du cours.

54 Les difficultés individuelles des étudiants préoccupent beaucoup le professeur.

55 Les exposés des travaux des étudiants occupent une place appropriée dans ce cours.

56 Ce cours me prépare adéquatement à ma future carrière.

57 Les objectifs d'apprentissage de ce cours sont formulés clairement.

58 La variété des travaux demandées aux étudiants est enrichissante.

59 Le professeur tient compte des habiletés individuelles d'apprentisssage.

\section{Satisfaction des étudiants par rapport à l'enseignement (QÉEC)}

60 J'estime que le professeur en tant qu'enseignant est

0 très mauvais 1 mauvais 2 acceptable 3 bon 4 excellent

61 J'estime que le professeur communique efficacement la matière

$\mathbf{0}$ presque jamais $\mathbf{1}$ rarement $\mathbf{2}$ parfois $\mathbf{3}$ souvent

4 presque toujours

62 J'estime que le professeur prépare bien ses cours

0 presque jamais $\mathbf{1}$ rarement $\mathbf{2}$ parfois $\mathbf{3}$ souvent

4 presque toujours 